\title{
Photodynamic therapy by conjugation of cell-penetrating peptide with fluorochrome
}

\author{
This article was published in the following Dove Press journal: \\ International Journal of Nanomedicine \\ 10 November 2017 \\ Number of times this article has been viewed
}

\section{Chul-Kyu Park',* \\ Yong Ho Kim ',* \\ Suhyun Hwangbo ${ }^{2}$ \\ Hoonsung $\mathrm{Cho}^{2}$}

'Department of Physiology, College of Medicine, Gachon University, Incheon, ${ }^{2}$ School of Materials Science \& Engineering, Chonnam National University, Gwangju, South Korea

*These authors contributed equally to this work
Correspondence: Hoonsung Cho School of Materials Science \& Engineering, Chonnam National University, 77 Yongbong-ro, Buk-gu, Gwangju, 6II86, South Korea

Tel +82625301717

Fax +82625301699

Email cho.hoonsung@jnu.ac.kr
Abstract: Photodynamic therapy (PDT) is a promising alternative therapy that could be used as an adjunct to chemotherapy and surgery for cancer, and works by destroying tissue with visible light in the presence of a photosensitizer (PS) and oxygen. The PS should restrict tissue destruction only to the tumor and be activated by light of a specific wavelength; both of these properties are required. Arginine-rich peptides, such as cell-penetrating peptides, have membrane-translocating and nuclear-localizing activities, which have led to their application in various drug delivery modalities. Protamine (Pro) is an arginine-rich peptide with membranetranslocating and nuclear-localizing properties. The reaction of an N-hydroxysuccinimide (NHS) ester of rhodamine (Rho) and clinical Pro was carried out in this study to yield RhoPro, and a demonstration of its phototoxicity, wherein clinical Pro improved the effect of PDT, was performed. The reaction between Pro and the NHS ester of Rho is a solution-phase reaction that results in the complete modification of the Pro peptides, which feature a single reactive amine at the N-terminal proline and a single carboxyl group at the C-terminal arginine. This study aimed to identify a new type of PS for PDT by in vitro and in vivo experiments and to assess the antitumor effects of PDT, using the Pro-conjugated PS, on a cancer cell line. Photodynamic cell death studies showed that the RhoPro produced has more efficient photodynamic activities than Rho alone, causing rapid light-induced cell death. The attachment of clinical Pro to Rho, yielding RhoPro, confers the membrane-internalizing activity of its arginine-rich content on the fluorochrome Rho and can induce rapid photodynamic cell death, presumably owing to lightinduced cell membrane rupture. PDT using RhoPro for HT-29 cells was very effective and these findings suggest that RhoPro is a suitable candidate as a PS for solid tumors.

Keywords: photodynamic therapy, rhodamine, protamine, endocytosis, cell-penetrating peptide

\section{Introduction}

Photodynamic therapy (PDT) is a clinically approved therapeutic method for the treatment of many malignant carcinomas. ${ }^{1}$ It involves the selective accumulation of a photosensitizer (PS) in tumor cells activated by irradiation of a specific wavelength, causing selective antitumor effects: direct cytotoxicity in tumor cells, involving the mitochondria-associated pathway and endoplasmic reticulum stress, due to the production of reactive singlet oxygen; devascularization of tumors; direct immune response by cytotoxic T cells against tumors; and elicitation of an immune response in the cells triggered by shutdown of the tumor vasculature, leading to local depletion of nutrients and oxygen in the tumor and causing secondary necrosis. ${ }^{2,3}$ These effects of PDT lead to several forms of cell death, including apoptosis, necrosis, and autophagy; different mechanisms account for these different forms of cell death. Apoptosis is the major process of cell death activated by PDT; however, cell death due to PDT generally occurs as a combination of these three mechanisms, and no single pathway of PDT 
leads to cell death. The factors determining mechanisms of cell death depend on various parameters: type and dose of PS, localization of PS, light dose, and oxygen concentration in the cell., ${ }^{4,5}$ Determination of major factors of cell death requires further study, but one factor postulated is the dosage of PS: high dose of PS leads to necrosis, while low dose of PS tends to activate apoptosis.

Since PDT was first used clinically in 1898, many of its advantages have been exploited. A combination of PDT and surgery, radiotherapy, or chemotherapy features low- or noninvasiveness, a low incidence of side effects, good compatibility with other treatments, fewer risks over repeated treatments, short treatment time, cost-effectiveness, and non-immunosuppression. ${ }^{6,7}$ However, there were also several drawbacks to PDT, preventing it from being used as a major treatment modality: dependence on certain types of PSs and light sources (wavelength, time exposure, pulse duration, and pulse frequency), low delivery accuracy of the PSs, and limited treatment depth. ${ }^{7,8}$ Therefore, to improve the efficiency of PDT and expand its applicability in the treatment of various cancers, the limitations regarding its clinical efficacy should be reduced. PDT requires light irradiation at a specific point in a tumor; therefore, monitoring of the tumor location is crucial for effective PDT with low side effects. ${ }^{9}$ Another limitation is that singlet oxygen has a very short half-life of less than $40 \mathrm{~ns}$ and a proliferation range of 10-20 nm; thus, effective delivery of the PS is required to enhance the availability of singlet oxygen in the cytoplasm and to direct it towards the organelles of tumor cells. ${ }^{10}$ To overcome the limitations of PDT, it is necessary to generate efficient methods for monitoring the accumulation of PSs and for delivering them to a selected site at a low dosage. We developed a new approach to the delivery of PSs by conjugation of a cell-penetrating peptide (CPP) with a typical fluorescent dye, rhodamine (Rho).

CPPs are interesting, as they can easily translocate through cellular membranes. ${ }^{11} \mathrm{CPP}$ typically contains 20-30 amino acid residues and is classified as amphipathic in nature, although cationic CPP has fewer amino acids with positively charged residues (arginine and lysine). ${ }^{12-14}$ CPPs offer an efficient way for drugs to pass easily through cellular membranes. Notably, CPPs rich in arginine penetrate cell membranes very readily. Arginine-rich peptides have membrane-translocating and nuclear-localizing sequences, ${ }^{15-18}$ which have led to their use in various drug delivery methods. ${ }^{19-21}$ Protamine (Pro), used to neutralize the anticoagulant effects of heparin, is a mixture of four similar arginine-rich peptides with membrane-translocating and nuclear-localizing properties. ${ }^{22}$ The advantage to using clinical Pro as a CPP is that the sequence of clinical Pro features a single reactive amine at the $\mathrm{N}$-terminal proline and a single reactive carboxyl group at the $\mathrm{C}$-terminal arginine providing simple steps for manipulation or modification of Pro by the addition of Rho. ${ }^{23}$

Therefore, in this study, we describe the reaction of an N-hydroxysuccinimide (NHS) ester of Rho and clinical Pro, yielding rhodamine-protamine (RhoPro), and demonstrate that clinical Pro confers phototoxicity on Rho. The fluorescent dye used for this study itself does not generate enough ROS to induce cell damage by light. However, conjugation of CPPs to the dye localizes the conjugates to specific subcellular locations via endocytosis, and the localized conjugates induce photodynamic effects when exposed to light. In addition, imaging of the fluorescent dye allows for tracking of the circulation and accumulation of the PSs in the body. We investigated cancer therapy with photodynamic effects, using CPPs conjugated with a fluorescent dye.

\section{Materials and methods Reagents and materials}

HT-29 colorectal adenocarcinoma cells were purchased from American Type Culture Collection (Manassas, VA, USA). 5-(and-6)-Carboxytetramethylrhodamine, succinimidyl ester (TAMRA), and LysoTracker Green were purchased from Invitrogen (Waltham, MA, USA), and Pro sulfate was purchased from App Pharmaceuticals (Schaumburg, IL, USA). SYTOX Blue was obtained from Molecular Probes (Eugene, OR, USA). A PD-10 column was purchased from GE Healthcare (Chicago, IL, USA), and dialysis cassettes were purchased from Pierce (Waltham, MA, USA). N,N-Dimethyl-4-nitrosoaniline (RNO) was purchased from Acros Organics (Pittsburgh, PA, USA), and Alexa Fluor ${ }^{\circledR} 488$ anti-mouse CD31 antibody was purchased from BioLegend (San Diego, CA, USA). For the cell cytotoxicity assay, MTT was purchased from Life Technologies (Carlsbad, CA, USA). Female BALB/c-Nu/Nu mice (6-week-old) were obtained from Orient Bio (Seongnam, South Korea) and maintained under specific pathogen-free conditions.

\section{Synthesis and purification of RhoPro}

Two milligrams of TAMRA (MW 430.45) was dissolved in $400 \mu \mathrm{L}$ of DMSO, and $15 \mathrm{mg}$ of Pro (in $1.5 \mathrm{~mL}$ of $0.85 \mathrm{M}$ $\mathrm{NaCl}$ as supplied, $\mathrm{MW} \sim 4,000 \mathrm{Da}$ ) was added along with $600 \mu \mathrm{L}$ of PBS. The reaction was carried out for $2 \mathrm{~h}$ at $25^{\circ} \mathrm{C}$. To saturate Pro with Rho, an additional $2 \mathrm{mg}$ of TAMRA was added to the reaction mixture, and the reaction process was repeated. The mixture was then purified through gel filtration using a PD-10 column and dialysis. The RhoPro products were characterized by high-performance liquid 
chromatography (HPLC) with absorbance maxima at 220 and $556 \mathrm{~nm}$ (C-18 column; gradient: 20\%-80\% B; eluent A $[0.1 \%$ TFA/water $]$ and eluent B [0.1\% TFA and 9.9\% water in acetonitrile], $15 \mathrm{~min}$ ) and matrix-assisted laser desorption/ ionization mass spectrometry (MALDI-MS).

\section{Cellular uptake of Rho and RhoPro}

Cellular uptake assays of RhoPro and Rho were conducted using 24 -well plates. HT-29 cells were seeded at $2 \times 10^{5}$ cells/well and incubated for $24 \mathrm{~h}$ before assaying for RhoPro and Rho. HT-29 cells were exposed to final concentrations of $10 \mu \mathrm{M}$ of RhoPro and Rho for the indicated durations. After incubation, cells were washed with Dulbecco's PBS (DPBS), and then dissociated (200 $\mu \mathrm{L}$ of $0.05 \%$ trypsin with $0.53 \mathrm{mM}$ EDTA, $10 \mathrm{~min}$ at $37^{\circ} \mathrm{C}$ ), pelleted $(1,500 \mathrm{rpm}, 5 \mathrm{~min})$, resuspended in $300 \mu \mathrm{L}$ of DPBS with $\mathrm{Ca}^{2+}, \mathrm{Mg}^{2+}$, and $1 \%$ fetal calf serum, and analyzed with a FACSCalibur flow cytometer. For confocal imaging, cells were stained with $500 \mathrm{nM}$ of LysoTracker, a green fluorescent lysosome-staining dye, for $30 \mathrm{~min}$ at RT. After incubation with the dye, cells were washed with DPBS three times and resuspended in the original medium. Images were obtained using a Zeiss LSM510 laser scanning confocal microscope.

\section{Cell death in response to the photodynamic effect of RhoPro}

To assess the cytotoxicity of RhoPro uptake, cells were seeded into 48 -well plates and incubated with $10 \mu \mathrm{M}$ of RhoPro and Rho for $2 \mathrm{~h}$ at $37^{\circ} \mathrm{C}$. After washing with PBS, cells were irradiated with light covering the entire area of the well and then stained with SYTOX Blue and/or Cy5.5 Annexin V. Cells were trypsinized, washed with PBS, and resuspended in $300 \mu \mathrm{L}$ of DPBS with $1 \%$ fetal calf serum, for flow cytometry. The photodynamic effect of RhoPro was determined with a dead cell-staining dye, SYTOX Blue. The power source was OSL2 (THORLABS) with a $150 \mathrm{~W}$ halogen lamp, and irradiation energy was measured with a PM100D optical power and energy meter (THORLABS). Light passed through 540/50-nm single-band filter (Semrock) and exposed a single well of 96 -well plate $\left(1.33 \mathrm{~cm}^{2}\right)$ at $40 \mathrm{~mW} / \mathrm{cm}^{2}$ for $12.5 \mathrm{~min}$; therefore, the irradiation energy of light reaching the cells was $30 \mathrm{~J} / \mathrm{cm}^{2}$. Images with and without light were taken at the boundaries of an irradiated area. Cells were incubated in 24-well plates, and the centers of the wells were illuminated with a $6.5-\mathrm{mm}$ diameter beam of light $\left(30 \mathrm{~J} / \mathrm{cm}^{2}\right)$. For visualizing the effect of PDT, time-lapse experiments with an LSM 510 confocal microscope were performed. Confocal excitation lasers were used as an irradiation source, and images were taken at 30-s intervals for $1 \mathrm{~h}$. The fluorescence intensities of RhoPro, Rho, and SYTOX Blue were obtained from the time-lapse experiments. One cell in each experiment was selected, and the mean intensity of the whole-cell body was measured using ZEN 2011 blue edition.

\section{RNO bleaching assay}

The generation of singlet oxygen was detected by the spectrophotometric measurement of RNO bleaching. Twenty micromolar Rho, RhoPro, and a mixture of Rho and Pro were mixed with $100 \mu \mathrm{M}$ of RNO and irradiated with light under the same conditions as in the photodynamic experiment.

\section{In vivo study}

All animal experiments were performed according to the regulations of the Animal Protection Act (Act number 10995) in accordance with protocols approved by the Chonnam National University Animal Research Committee, which also approved this research. Six- or eight-week-old female nude mice were purchased from Orient Bio, and the animals received $10 \mathrm{mg} /$ $\mathrm{kg}$ of RhoPro intravenously followed by intravenous injection of $10 \mu \mathrm{g}$ of Alexa Fluor 488 anti-mouse CD31 antibody 90 min after RhoPro administration. Twenty minutes after CD31 injection, the mice were euthanized and organs harvested. The mean fluorescence intensity of the organs was obtained from the analysis of fluorescence images of the 1-mm-thick organ slices, obtained using a Kodak Whole-Mouse Image Station. For histological analysis of the accumulation of RhoPro in tumors, the tumors were embedded in OCT compound and sliced at $-20^{\circ} \mathrm{C}$, using a cryostat. For in vivo PDT study, $1 \times 10^{7} \mathrm{HT}-29$ human colorectal cancer cells were implanted in the mice on both sides of the back. After 1 week, the mice were anesthetized using $2 \%$ isoflurane in $1 \mathrm{~L} / \mathrm{min} \mathrm{O}^{2}$, and received direct intratumoral injections of $10 \mathrm{mg} / \mathrm{kg}$ of RhoPro and Pro into each side of the tumor, followed by irradiation with $50 \mathrm{~J} / \mathrm{cm}^{2}$ for PDT. Three mice were used for each injection. The tumor size was measured every alternate day, and the second administration of PS and PDT was carried out on day 3. The two-way ANOVA (GraphPad Prism7) was used to compare the difference between Pro-treated group and RhoPro-treated group.

\section{Results and discussion}

\section{Synthesis and characterization of RhoPro}

The reaction between clinical Pro and the NHS ester of Rho is a solution-phase reaction that occurs at the N-terminal proline and results in complete modification of the four Pro peptides. Pro is a series of four similar, arginine-rich peptides, with a single reactive amine at the $\mathrm{N}$-terminal proline and a single carboxyl group at the $\mathrm{C}$-terminal arginine, which enable controlling the ratio of Pro to Rho. Since no Pro remains after reaction with the NHS ester of Rho, separation 
of Rho (MW 527.53) and RhoPro (MW $\approx 4,500 \mathrm{Da})$ was accomplished through readily scalable, size-dependent separation methods (gel filtration and dialysis). As indicated in Figure 1A, NHS ester of 5-(and-6)-carboxytetramethylrhodamine was reacted with Pro two times to consume all Pro and enable purification by dialysis to eliminate the unreacted Rho. Figure 1B shows a reverse-phase HPLC plot verifying the absence of free Pro in the unpurified reaction mix and the absence of free Rho after purification. The two peaks of RhoPro in the HPLC plot are shown to have identical masses through MALDI-MS (data not shown). The upper mass spectrum of MALDI-MS (Figure 1C) shows RhoPro after completing the reaction, and the lower mass spectrum corresponds to the product in the middle of reaction (peaks 1-4 are Pro; peaks 5-8 are RhoPro). This indicates that all four peptides of Pro reacted with Rho, undergoing an increase in mass of $412.78 \pm 0.15 \mathrm{Da}$ (for example, peak 5 of RhoPro has a mass of 4,479 , which matches the sum of the mass of Pro peak 1 [4,066] and the mass of Rho [413]). The absorption spectra confirmed RhoPro synthesis, showing precisely the peaks of Pro and Rho (Figure 1D).

\section{Cellular uptake of RhoPro}

Cellular uptake experiments comparing RhoPro with Rho were performed on HT-29 cells to study uptake kinetics and distribution. We incubated HT-29 cells with $10 \mu \mathrm{M}$ RhoPro or Rho for up to $2 \mathrm{~h}$ at $37^{\circ} \mathrm{C}$ and measured cell fluorescence by flow cytometry. Dual-wavelength fluorescenceactivated cell sorting (FACS) scattergrams showed uptake as RhoPro fluorescence and toxicity as SYTOX fluorescence (Figure 2A). The dead cell-staining dye, SYTOX Blue, penetrates cells with compromised plasma membranes and fluoresces upon binding DNA. No change in the intensity of SYTOX Blue indicates the uptake of RhoPro without cytotoxicity. The time course of RhoPro uptake measured by a single channel FACS $(0,5,15,30,60$, and $120 \mathrm{~min})$ in Figure 2B showed that RhoPro interacted with cells slowly, unlike Rho (all scattergrams regarding uptake of RhoPro and Rho are shown in Figure S1). Figure 2C shows the median intracellular fluorescence of the cells as a function of time. Cells treated with only Rho were saturated immediately because Rho is a membrane-permeable fluorochrome that is able to passively diffuse through the membrane; RhoPro

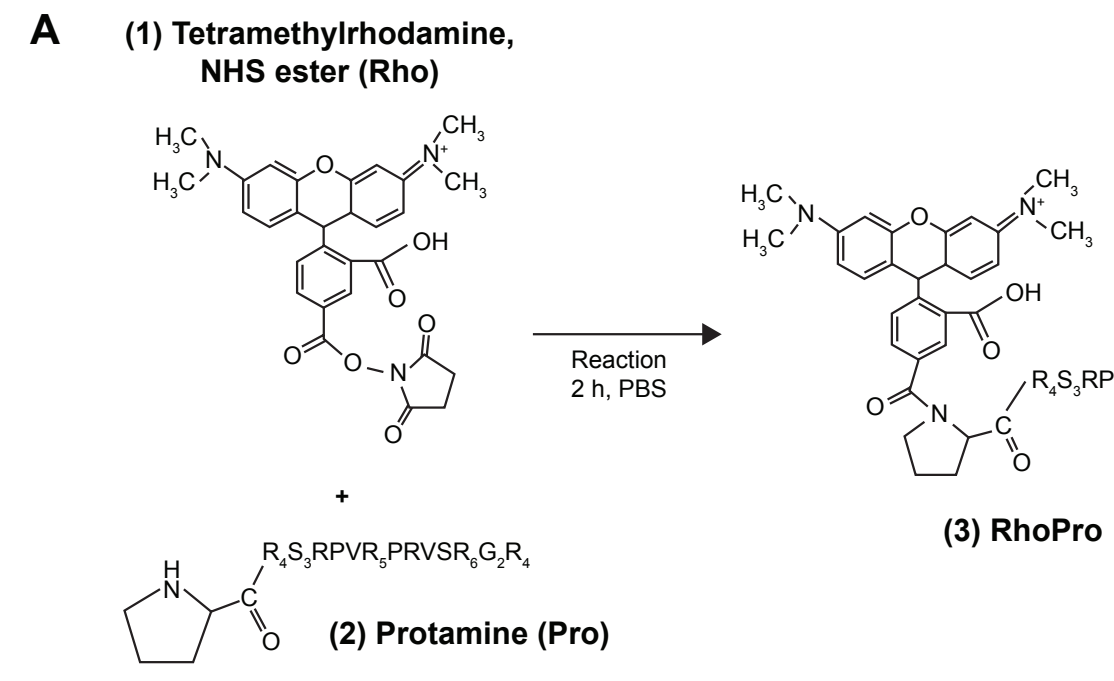

B

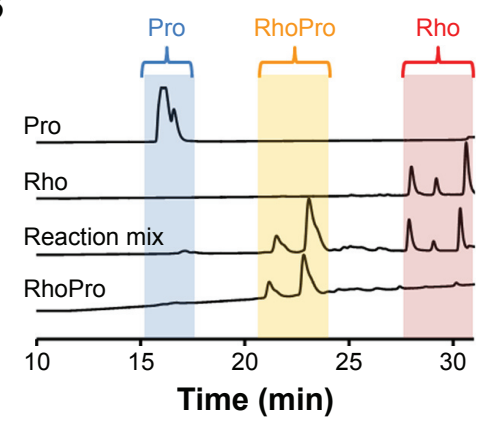

C

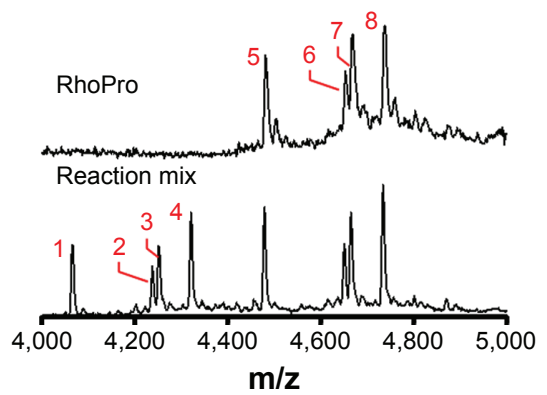

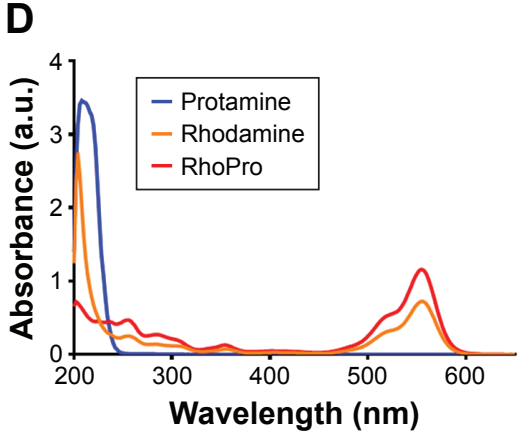

Figure I Synthesis and purification of RhoPro. (A) An NHS ester of Rho (I) reacts with N-terminal proline of Pro (2) to yield RhoPro (3). (B) High-performance liquid chromatography chromatograms show the separation of RhoPro from Pro and Rho. (C) Mass spectrometry showing four Pro components and four RhoPros generated by reaction of the N-terminal prolines as shown in A. (D) Absorption spectra of Pro, Rho, and purified RhoPro. Peaks I-4 are Pro; peaks 5-8 are RhoPro.

Abbreviations: NHS, N-hydroxysuccinimide; Pro, protamine; Rho, rhodamine; RhoPro, rhodamine-protamine. 
A

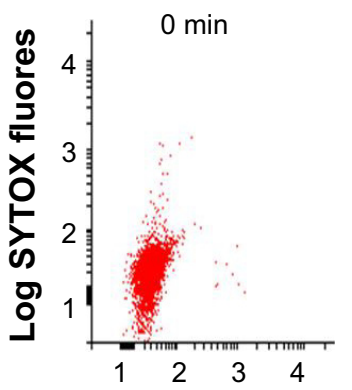

4

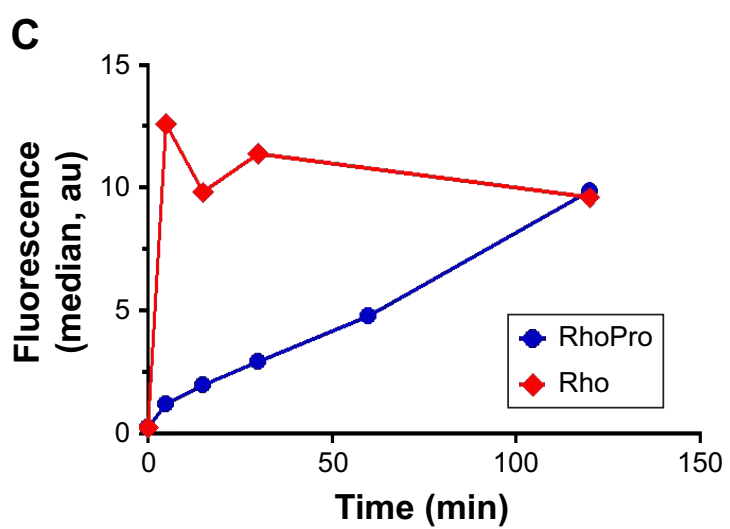

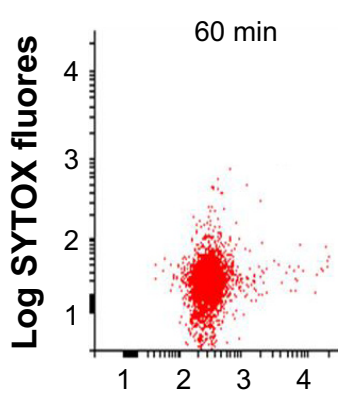

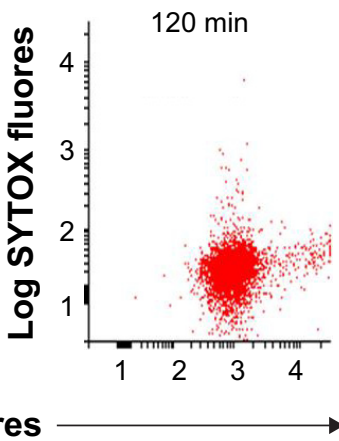

D
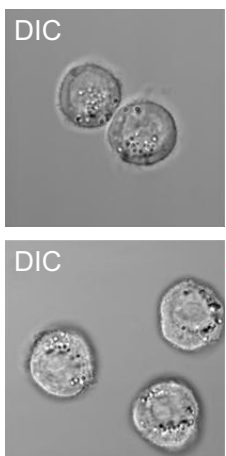

B

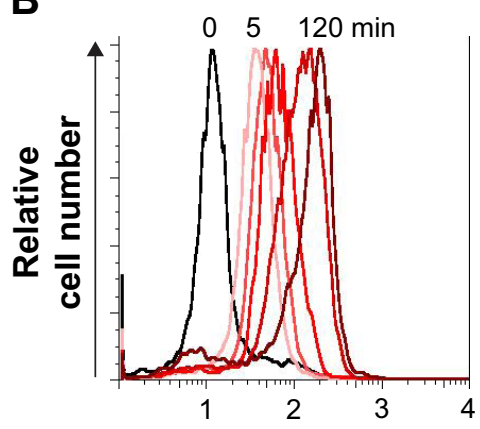

Log RhoPro fluores

Figure 2 Uptake of RhoPro by HT-29 cells observed through FACS and the endosomal intracellular localization of RhoPro by fluorescence microscopy. (A) Dual-wavelength FACS scattergrams showing uptake of RhoPro and SYTOX Blue. (B) Time course of RhoPro uptake by single-channel FACS (0, 5, I5, 30, 60, and I 20 min). Black line $=0$ min, pink line $=5 \mathrm{~min}$, lightest red line $=15 \mathrm{~min}$, medium red line $=30 \mathrm{~min}$, dark red line $=60 \mathrm{~min}$, darkest red line $=120 \mathrm{~min}$. $(\mathbf{C})$ Median cell fluorescence from $(\mathbf{B})$ plotted as a function of incubation time. Rho is rapidly internalized, while RhoPro is slowly internalized. (D) Intracellular localization of RhoPro on fluorescence microscopy. Rho fluorescence appears to be diffused throughout the cell. RhoPro colocalizes with LysoTracker Green (LTG), a marker of lysosomes. Magnification: 60x oil objective.

Abbreviations: DIC, differential interference contrast; FACS, fluorescence-activated cell sorting; fluores, fluorescence; Rho, rhodamine; RhoPro, rhodamine-protamine.

was taken up slowly, reaching maximum fluorescence after $2 \mathrm{~h}$ of incubation because RhoPro is translocated into the cells by endocytosis, similar to the internalization of the CPP Pro. Those different internalization processes led to different distribution patterns (Figure 2D). Figure 2D (upper) shows a uniform distribution of Rho through the cell, but RhoPro enters the cell as endocytic vesicles confined to the lysosomes (Figure 2D, lower). RhoPro colocalizes with the lysosomal marker LysoTracker Green, indicating that RhoPro has been internalized by the endocytic pathway and accumulated in lysosomes.

\section{PDT effect on cell death in response to RhoPro}

We performed photodynamic cell death studies by flow cytometry and fluorescence microscopy. The radiation source was filtered with a 540/50-nm single-band filter because the maximum efficacy of photodynamic treatment has been achieved at the wavelength of the absorbance maxima of the PS. ${ }^{24}$ Flow cytometry showed cell death associated with RhoPro treatment (Figure 3A). Without any compound and with Rho and Pro alone, there was no significant change in the population of dead cells irrespective of light application; however, when the RhoPro was irradiated, the percentage of dead cells increased by $36.2 \%$. Figure 3B shows the comparison of the populations of dead cells obtained, through FACS. In addition, we observed photodynamic-induced cell death using SYTOX Blue and a fluorescence microscope (Figure 3C and D). The irradiation beam was adjusted to illuminate the center of the cell-implanted plate well, and images were taken on the boundary of the beam circle. Cells treated with RhoPro showed PDT-induced cell death confirmed by SYTOX Blue in the light-exposed area (Figure 3D), but no phototoxicity was observed in treatments with Rho only (Figure 3C). Populations in the circle show SYTOX Bluepositive dead cells. As shown in Figure 3D, decolorization of the cells with RhoPro was observed upon irradiation, while Rho fluorescence exhibited no change after irradiation.

To further examine the effect of PDT in vitro, we conducted time-lapse confocal experiments to visualize the change in cellular morphology and analyze the intensity of the fluorescent probes during photodynamic treatment (Figure 4). Confocal excitation lasers were used as an irradiation source, and images were obtained at 30-s intervals 

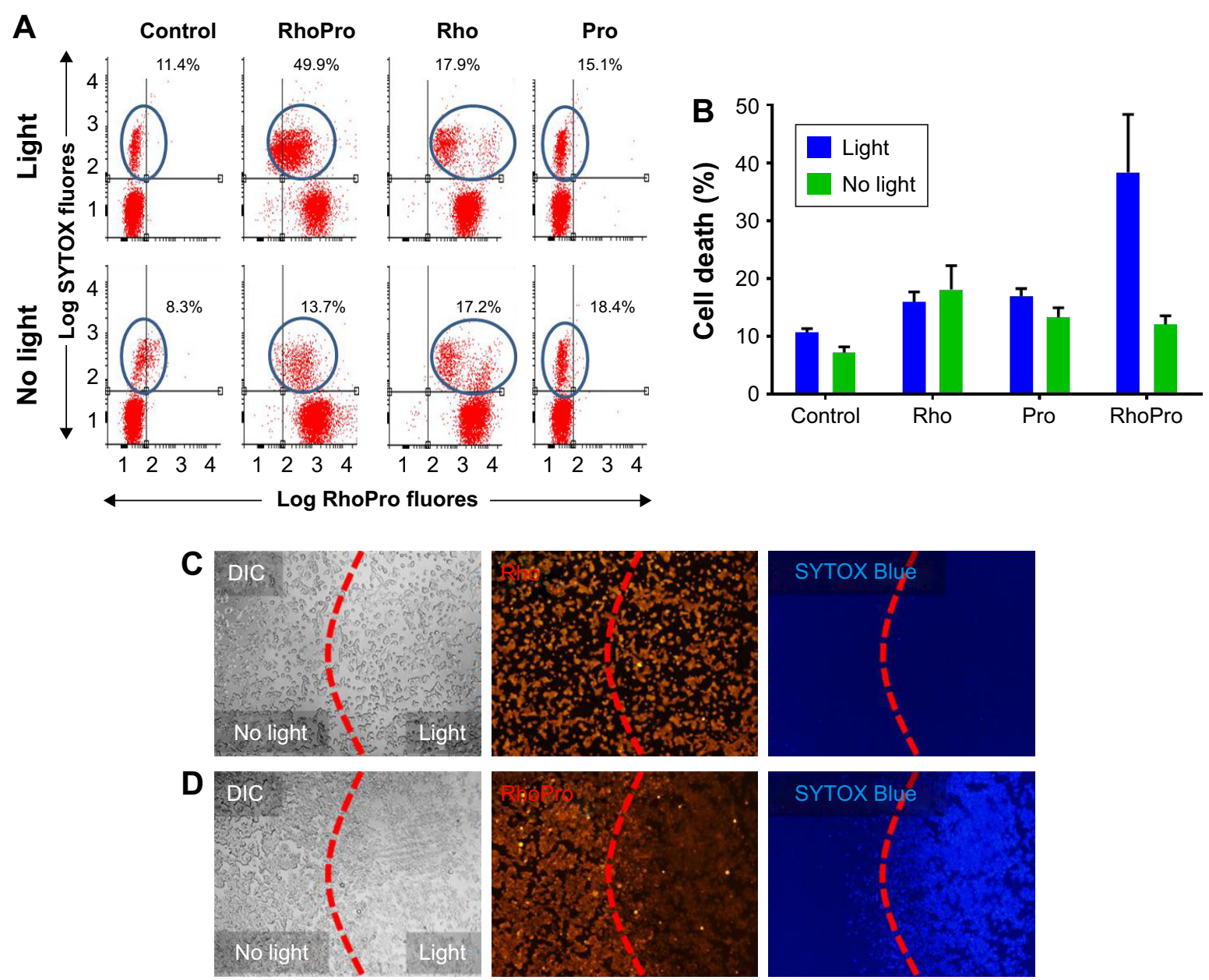

Figure 3 Phototoxicity of RhoPro-loaded HT-29 cells. (A) Dual-channel FACS scattergrams show RhoPro uptake (x-axis) and cell death ( $y$-axis) with light and without light. Control groups indicate a role of irradiation only without any uptake. The blue circles indicate the positive fluorescence of the dead cell staining. (B) Data from the selected area of scattergrams are plotted as median fluorescence. The combination of light plus RhoPro uptake results in cell death. Microscopic imaging of light-induced cell death in (C) Rho- or (D) RhoPro-loaded cells. Cells were imaged by phase-contrast (DIC) microscopy with part of the field of view subjected to light. As shown in D, fluorescence of RhoPro decreased (bleached) while SYTOX Blue penetrated the illuminated cells. Magnification: I0× objective. Left side of the red dashed lines indicates regions with no light, while right side indicates regions with light.

Abbreviations: DIC, differential interference contrast; FACS, fluorescence-activated cell sorting; fluores, fluorescence; Pro, protamine; Rho, rhodamine; RhoPro, rhodamine-protamine.

for $1 \mathrm{~h}$. Cells were intact and the lysosomal localization of RhoPro was distinct at the beginning of the experiment (Figure 4A), while the image taken at the end of the experiment showed cytoplasmic extrusion in the form of large bubbles, blebbing of cytoplasmic organelles and the nucleus, and the rupture of the plasma membrane followed by SYTOX Blue nucleic staining (Figure 4B). To quantify Rho fluorescence bleaching and staining time during the PDT process, we measured the whole-cell fluorescence intensity of RhoPro, Rho, and SYTOX Blue over time (Figure 4C). The mean intensity of Rho began to decrease after irradiation, and bleaching was completed within $15 \mathrm{~min}$. The bleaching rate of Rho was slower than that of RhoPro. Intensity of SYTOX Blue in cells with Rho was marginally detected after $25 \mathrm{~min}$, but did not increase as much as the intensity in cells with RhoPro implicated in reversible apoptosis, which corresponds to the cell images in Figure 4B. However, cells with RhoPro showed morphological changes typical of necrosis (Figure 4B) and an increase in the intensity of SYTOX Blue dead cell-staining dye by $25 \mathrm{~min}$ (Figure 4C).

\section{RNO bleaching assay}

To determine of the role of Pro in the photodynamic effects of RhoPro, RNO bleaching experiments were performed to measure the generation of ${ }^{1} \mathrm{O}_{2}$. Rho is commonly used as a staining dye for molecular biology assays, but also used as a PS because of the high quantum yield $\left(\Phi_{f}>0.9\right)$ and incidentally low ROS yield. Assessment of phototoxicity, using Rho, revealed no cell death up to $100 \mathrm{~J} / \mathrm{cm}^{2} .{ }^{25}$ Figure 4D shows the loss of RNO absorbance by irradiation at indicated time points in the presence of RhoPro, Rho + Pro, and 
A
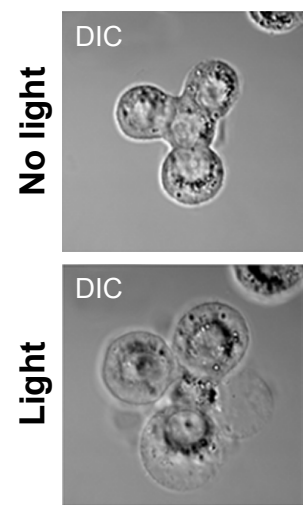
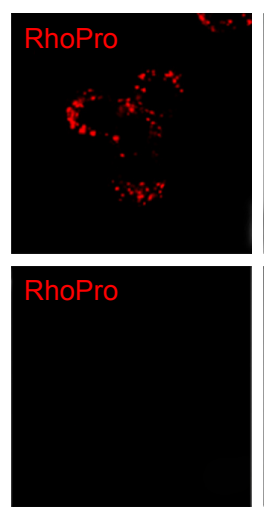
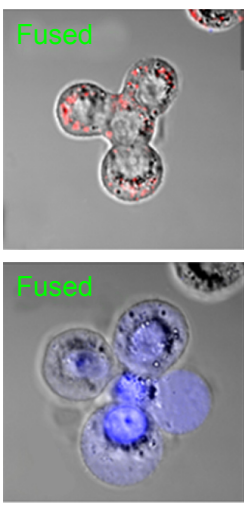
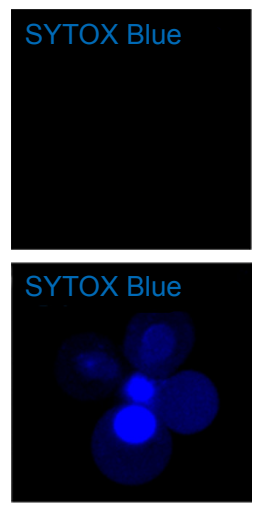

B
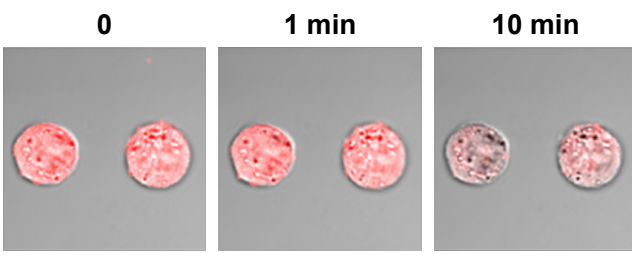

$20 \min$

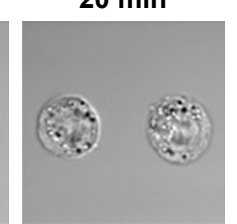

$30 \mathrm{~min}$
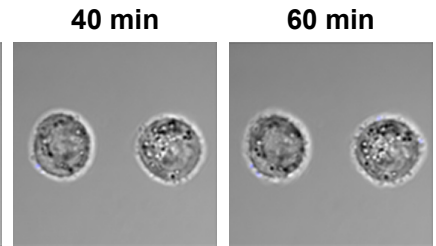

\section{DIC and Rho fluores}
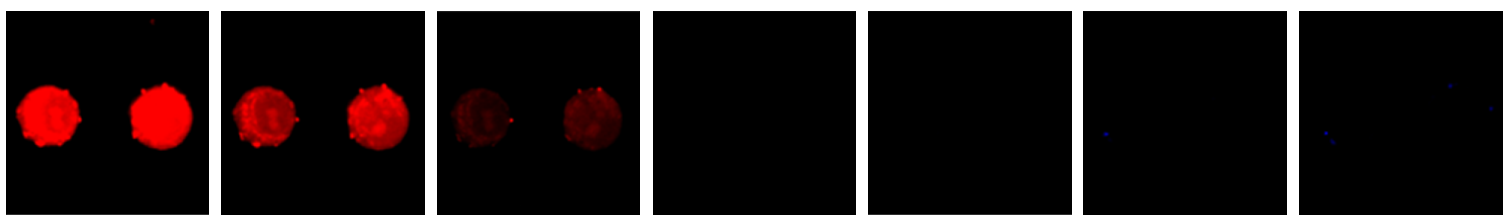

Rho and SYTOX fluores
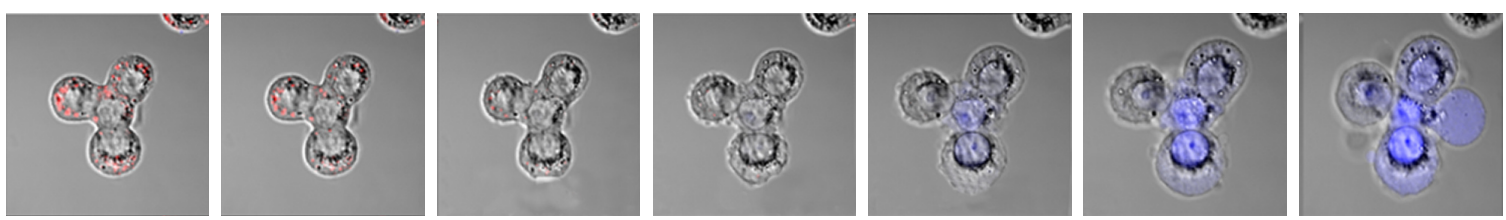

\section{DIC, RhoPro, and SYTOX fluores}
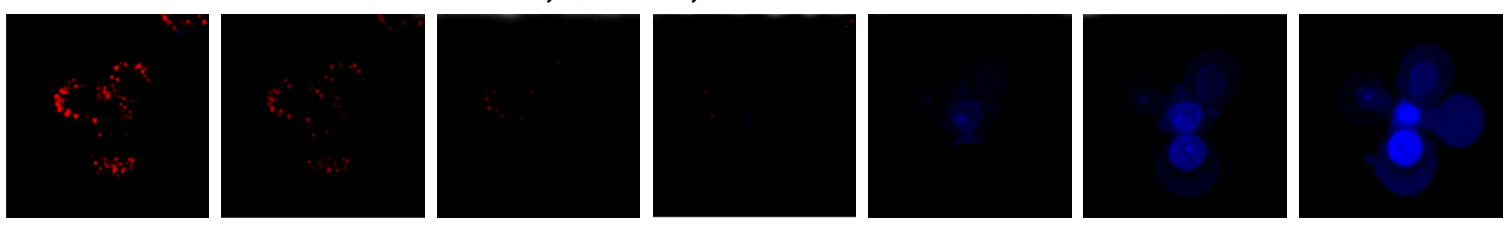

RhoPro and SYTOX fluores

C

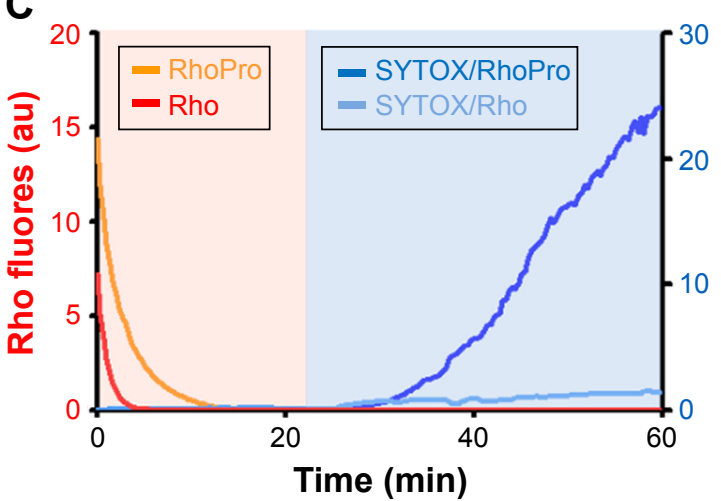

D

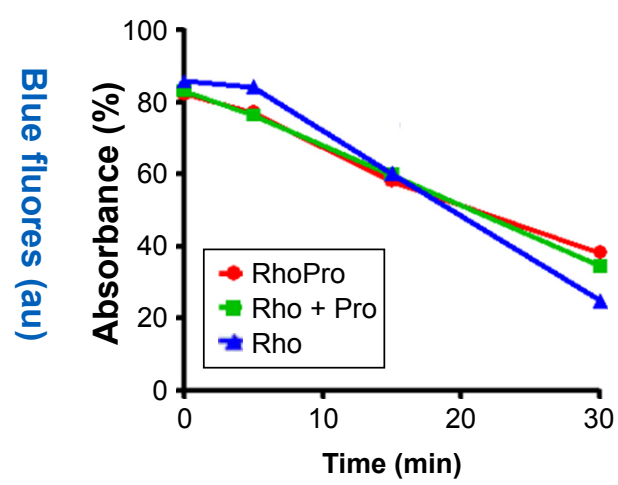

Figure 4 Kinetics of light-induced cell death in RhoPro-loaded cells. (A) With light, RhoPro fluorescence bleaches and induces RhoPro-loaded cells to take up SYTOX Blue. (B) Kinetics of light-induced RhoPro bleaching and SYTOX entry. Top two frames: DIC and Rho fluorescence shown as a function time of exposure to light. Bottom two frames: light induced SYTOX uptake. Magnification: $60 \times$ oil objective. (C) Time course for RhoPro and Rho bleaching and SYTOX uptake. RhoPro bleaches first, and cell death occurs as SYTOX entry commences. Additionally, the bleaching of rhodamine (Rho)-loaded cells and lack of SYTOX penetration are shown. (D) RNO bleaching assay showing generation of free oxygen radicals.

Abbreviations: DIC, differential interference contrast; fluores, fluorescence; Pro, protamine; Rho, rhodamine; RhoPro, rhodamine-protamine; RNO, N,N-dimethyl-4nitrosoaniline. 
Rho alone. There was no significant difference in ${ }^{1} \mathrm{O}_{2}$ yield irrespective of the conjugation of Rho with Pro, implying that the photodynamic effects of RhoPro occur by specific localization of Rho in a cell body, and not by photochemical changes from conjugating. This finding suggests that Pro conjugation converts any fluorochrome, not yielding a useful amount of ${ }^{1} \mathrm{O}_{2}$, into a PS for PDT. However, it is still important that the fluorochrome generates singlet oxygen per se. It has been reported that the endosomally internalized fluorescein-labeled CPPs were redistributed into the cytoplasm on irradiation, but that the cells remained intact and showed no phototoxicity. ${ }^{26}$

\section{Lysosomal rupture by PDT}

Lysosomes are cytoplasmic organelles that contain various types of hydrolytic enzymes and maintain acidic conditions $(\mathrm{pH} \leq 5)$, serving in the digestion of unnecessary wastes and playing an important role in programmed cell death. Studies have reported that PDT triggers lysosomal membrane permeabilization through release of cathepsins and induces lysosomal cell death. ${ }^{27,28}$ As mentioned in Figure 2D, RhoPro was introduced into the cell and localized in lysosomes by endocytosis, and this is clearly shown in Figure 5A. Accumulation of RhoPro in lysosomes was verified by co-staining with the lysosome staining dye LysoTracker Green. Most of the lysosomes emitted vivid fluorescence and had clear shape before irradiation (yellow arrows in Figure 5A). In contrast, irradiation induced the direct rupture of lysosomes and fluorescence of both RhoPro and LysoTracker Green diffused in the cytoplasm (Figure 5B), which in turn may have induced necrotic cell death. The major phenomenon induced by PDT is apoptotic cell death participating directly in cytotoxicity, vascular damage, and activation of the immune system, while necrosis induces cytotoxicity and vascular damage directly. However, it has been reported that cytotoxicity via necrosis led to more cell damage beyond the scope of rescue mechanisms than did apoptosis at the same dosage. ${ }^{29}$ Therefore, necrosis induced by RhoPro would enhance the efficacy of PDT with a low dose of PS and, in turn, cause fewer side effects.

\section{In vivo PDT of RhoPro}

Pro is used to prevent anticoagulant effects by binding negatively charged heparin. We therefore postulate that Pro would bind to endothelial cells because both heparin and endothelial cells have the same sulfate structure and strong negative charges. ${ }^{30}$ To evaluate the distribution of RhoPro in the body, we administered $10 \mathrm{mg} / \mathrm{kg}$ (intravenous) injections of RhoPro (Figure 6). One-millimeter-thick tissue sections were prepared, and the fluorescence of samples was measured. RhoPro accumulated in tissues lined with hepatic endothelial cells. The major organs exhibiting RhoPro accumulation in mice
A

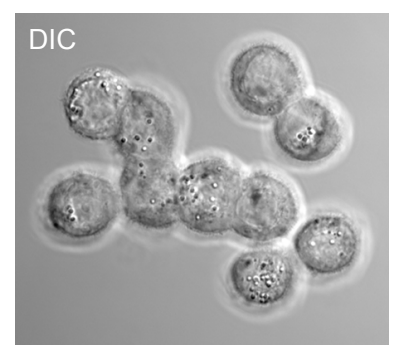

B

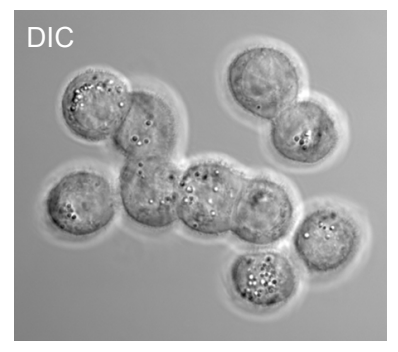

\section{Before irradiation}
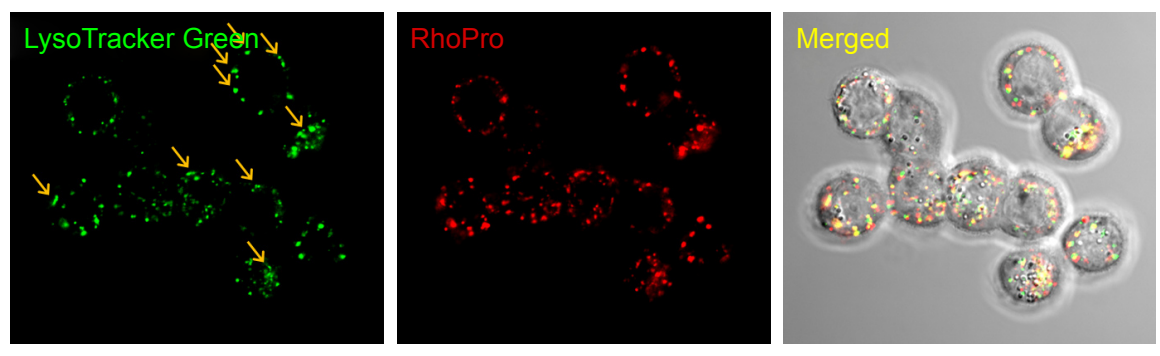

After irradiation
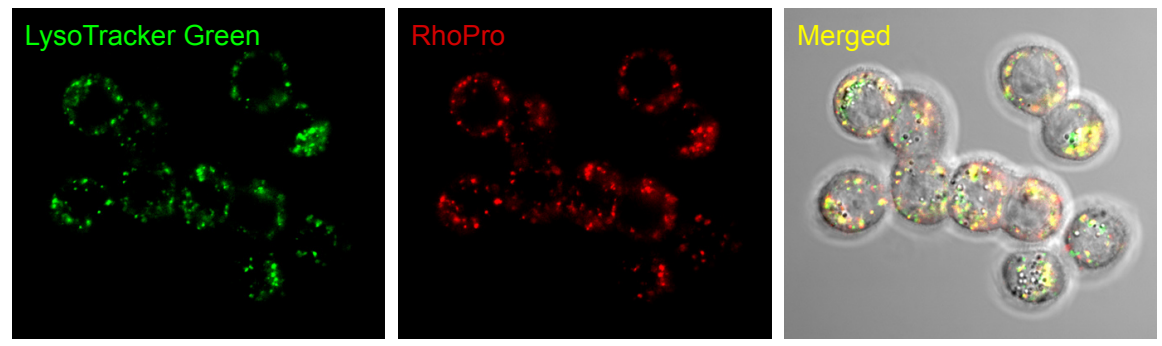

Figure $\mathbf{5}$ Lysosomal rupture induced by the photodynamic effect of RhoPro. (A) Internalized RhoPro was accumulated in lysosomes co-stained with the lysosome marker, LysoTracker Green. Yellow arrows indicate intact lysosomes. (B) After irradiation, lysosomal rupture occurred, and the fluorescence of LysoTracker Green and RhoPro decreased and appeared to diffuse in the cytoplasm. Magnification: 60× oil objective.

Abbreviations: DIC, differential interference contrast; RhoPro, rhodamine-protamine. 

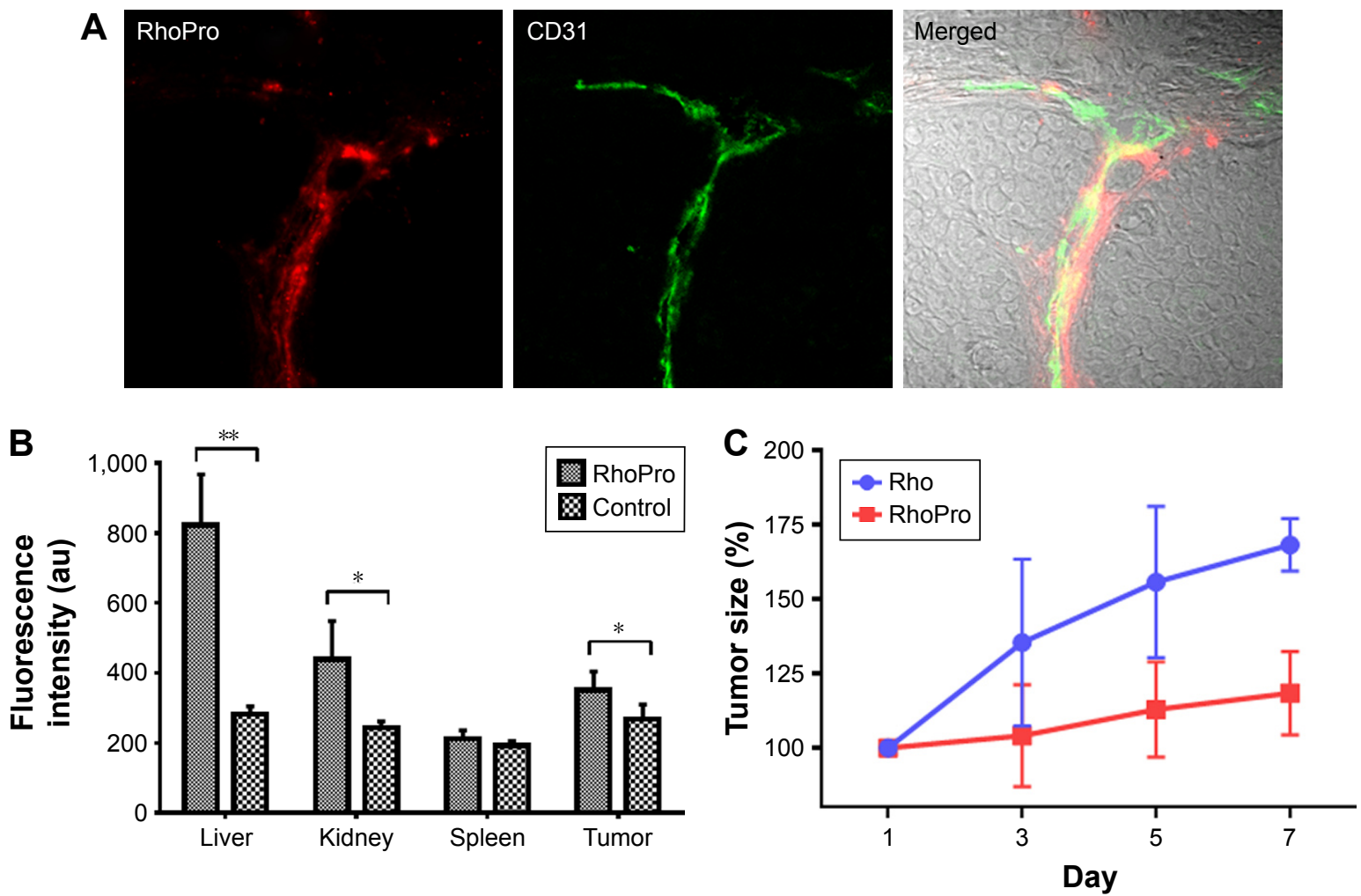

Figure 6 The in vivo photodynamic effect of RhoPro in tumor xenograft models. (A) Accumulation of RhoPro in tumor blood vessels confirmed with Alexa Fluor ${ }^{\circledR} 488$ anti-mouse CD3 I antibody. Magnification: 40× objective. (B) Biodistribution of RhoPro in HT-29 tumor-bearing mouse. The significant differences are shown as *P $<0.05$ and $* * P<0.01$. (C) In vivo PDT of RhoPro compared with that of Rho. PDT by RhoPro suppressed the tumor growth rate.

Abbreviations: PDT, photodynamic therapy; Rho, rhodamine; RhoPro, rhodamine-protamine.

were the liver and kidneys, including tumors (Figure 6B). Immunohistofluorescence analysis confirmed the capability of RhoPro to bind to endothelial cell-lined interior surfaces of blood vessels in tumors by co-staining with fluorochromelabeled CD31 antibodies (Figure 6A). The in vivo photodynamic effects of RhoPro were determined using an HT-29 subcutaneous mouse xenograft tumor model for consistency with in vitro experiments and for a better understanding of the biology of xenograft-human tumors than that of subcutaneous mouse tumors. ${ }^{31}$ Figure $6 \mathrm{C}$ shows the size changes of the tumor during PDT treatment. The tumor size of each mouse was considered $100 \%$ before PDT and measured every alternate day. Tumors treated with Rho increased to $68 \%$ in size after 7 days, while RhoPro significantly suppressed the increase in tumor size to $18 \%(P<0.0005)$.

\section{Conclusion}

We found a new type of PS by conjugating Pro with Rho. Photodynamic cell death studies showed that with no treatment and with Rho and Pro alone, there was no significant change in the population of dead cells irrespective of light application; however, when the RhoPro was irradiated, the percentage of dead cells increased by $36.2 \%$. In addition, using SYTOX Blue, a dead cell-staining dye, cells treated with RhoPro showed PDT-induced cell death in the exposed area, but no phototoxicity was observed on treatment with only Rho. Cells with RhoPro showed morphological changes typical of necrosis and increases of intensity of SYTOX Blue over time. The in vivo results confirmed the photodynamic effects of RhoPro on the tumors. RhoPro is simple to synthesize and purify, and can serve as a PS to confer photodynamic effects on fluorochromes, yielding low levels of ROS.

\section{Acknowledgment}

This study was supported by the Basic Science Research Program through the National Research Foundation of Korea (NRF) funded by the Ministry of Education (2015R1D1A1A01059289).

\section{Disclosure}

The authors report no conflicts of interest in this work.

\section{References}

1. Mroz P, Yaroslavsky A, Kharkwal GB, Hamblin MR. Cell death pathways in photodynamic therapy of cancer. Cancers. 2011;3(2):2516-2539.

2. Agostinis P, Berg K, Cengel KA, et al. Photodynamic therapy of cancer: an update. CA Cancer J Clin. 2011;61(4):250-281. 
3. Henderson BW, Dougherty TJ. How does photodynamic therapy work? Photochem Photobiol. 1992;55(1):145-157.

4. Buytaert E, Dewaele M, Agostinis P. Molecular effectors of multiple cell death pathways initiated by photodynamic therapy. Biochim Biophys Acta. 2007;1776(1):86-107.

5. Piette J. Signalling pathway activation by photodynamic therapy: NF- $\kappa \mathrm{B}$ at the crossroad between oncology and immunology. Photochem Photobiol Sci. 2015;14:1510-1517.

6. Patel LN, Wang J, Kim KJ, Borok Z, Crandall ED, Shen WC. Conjugation with cationic cell-penetrating peptide increases pulmonary absorption of insulin. Mol Pharm. 2009;6(2):492-503.

7. Hayley P, Stamati I, Yahioglu G, Butt M, Deonarain M. Antibodydirected phototherapy (ADP). Antibodies. 2013;2(2):270-305.

8. Calin MA, Parasca SV. Photodynamic therapy in oncology. J Optoelectron Adv Mater. 2006;8(3):1173-1179.

9. Allison RR, Downie GH, Cuenca R, Hu X-H, Childs CJH, Sibata CH. Photosensitizers in clinical PDT. Photodiagn Photodyn Ther. 2004; 1(1):27-42.

10. Guo M, Mao H, Li Y, et al. Dual imaging-guided photothermal/ photodynamic therapy using micelles. Biomaterials. 2014;35(16): 4656-4666.

11. Zheng Y, Yin G, Le V, et al. Photodynamic-therapy activates immune response by disrupting immunity homeostasis of tumor cells, which generates vaccine for cancer therapy. Int J Biol Sci. 2016;12(1):120-132.

12. Patel LN, Zaro JL, Shen WC. Cell penetrating peptides: intracellular pathways and pharmaceutical perspectives. Pharm Res. 2007;24(11): 1977-1992.

13. Zaro JL, Shen W-C. Cationic and amphipathic cell-penetrating peptides (CPPs): their structures and in vivo studies in drug delivery. Front Chem Sci Eng. 2015;9(4):407-427.

14. Madani F, Lindberg S, Langel, et al. Mechanisms of cellular uptake of cell-penetrating peptides. J Biophys. 2011;2011:414729.

15. Futaki $\mathrm{S}$, Goto $\mathrm{S}$, Sugiura Y. Membrane permeability commonly shared among arginine-rich peptides. J Mol Recognit. 2003;16(5):260-264.

16. El-Sayed A, Futaki S, Harashima H. Delivery of macromolecules using arginine-rich cell-penetrating peptides: ways to overcome endosomal entrapment. AAPS J. 2009;11(1):13-22.

17. Lindgren $M$, Langel $U$. Classes and prediction of cell-penetrating peptides. Methods Mol Biol. 2011;683:3-19.
18. Milletti F. Cell-penetrating peptides: classes, origin, and current landscape. Drug Discov Today. 2012;17(15-16):850-860.

19. Fonseca SB, Pereira MP, Kelley SO. Recent advances in the use of cellpenetrating peptides for medical and biological applications. Adv Drug Deliv Rev. 2009;61(11):953-964.

20. Gillmeister MP, Betenbaugh MJ, Fishman PS. Cellular trafficking and photochemical internalization of cell penetrating peptide linked cargo proteins: a dual fluorescent labeling study. Bioconjug Chem. 2011; 22(4):556-566.

21. Torchilin VP. Tat peptide-mediated intracellular delivery of pharmaceutical nanocarriers. Adv Drug Deliv Rev. 2008;60(4):548-558.

22. Reynolds F, Weissleder R, Josephson L. Protamine as an efficient membrane-translocating peptide. Bioconjug Chem. 2005;16(5): $1240-1245$.

23. Liang JF, Yang VC, Vaynshteyn Y. The minimal functional sequence of protamine. Biochem Biophys Res Commun. 2005;336(2):653-659.

24. Allison AC, Magnus IA, Young MR. Role of lysosomes and of cell membranes in photosensitization. Nature. 1966;209(5026):874-878.

25. Shea CR, Chen N, Wimberly J, Hasan T. Rhodamine dyes as potential agents for photochemotherapy of cancer in human bladder carcinoma cells. Cancer Res. 1989;49(14):3961-3965.

26. Maiolo JR, Ottinger EA, Ferrer M. Specific redistribution of cellpenetrating peptides from endosomes to the cytoplasm and nucleus upon laser illumination. J Am Chem Soc. 2004;126(47):15376-15377.

27. Boya P, Andreau K, Poncet D, et al. Lysosomal membrane permeabilization induces cell death in a mitochondrion-dependent fashion. J Exp Med. 2003;197(10):1323-1334.

28. Boya P, Kroemer G. Lysosomal membrane permeabilization in cell death. Oncogene. 2008;27(50):6434-6451.

29. Nagata S, Obana A, Gohto Y, Nakajima S. Necrotic and apoptotic cell death of human malignant melanoma cells following photodynamic therapy using an amphiphilic photosensitizer, ATX-S10(Na). Lasers Surg Med. 2003;33(1):64-70.

30. Lever R, Page CP. Novel drug development opportunities for heparin. Nat Rev Drug Discov. 2002;1(2):140.

31. Silva Zenildo S, Bussadori Sandra K, Fernandes K, Porta S, Huang Y-Y, Hamblin Michael R. Animal models for photodynamic therapy (PDT). Biosci Rep. 2015;35(6):e00265. 


\section{Supplementary material}

A
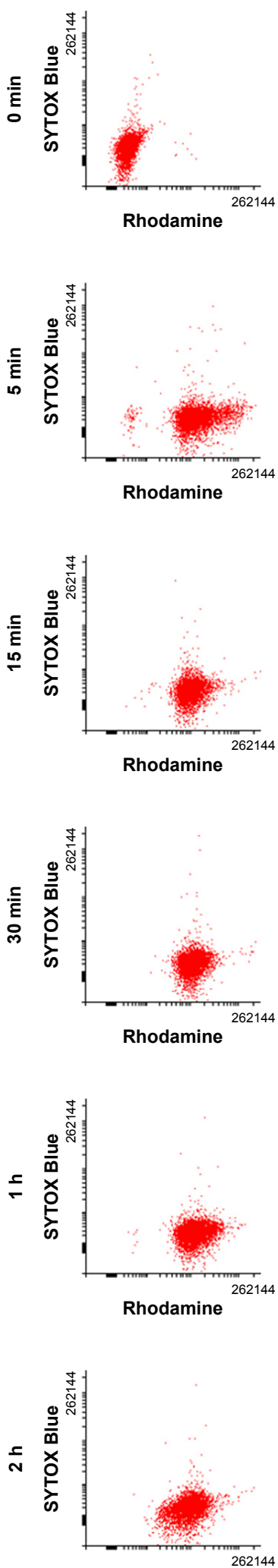

Rhodamine
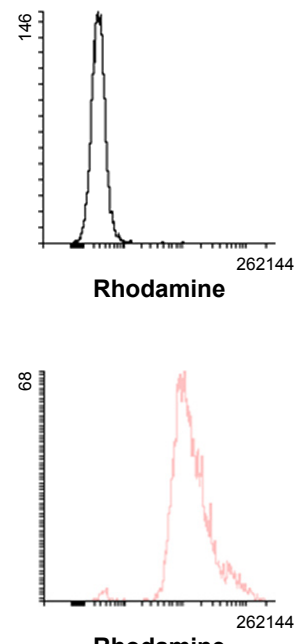

Rhodamine
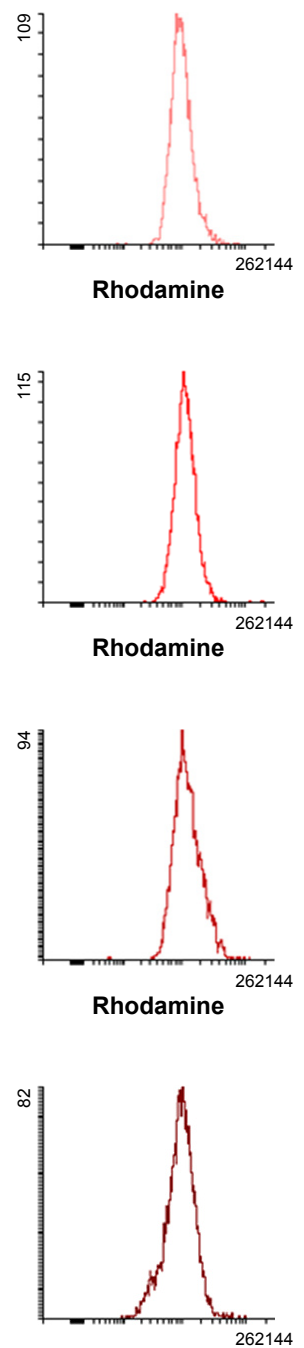

Rhodamine
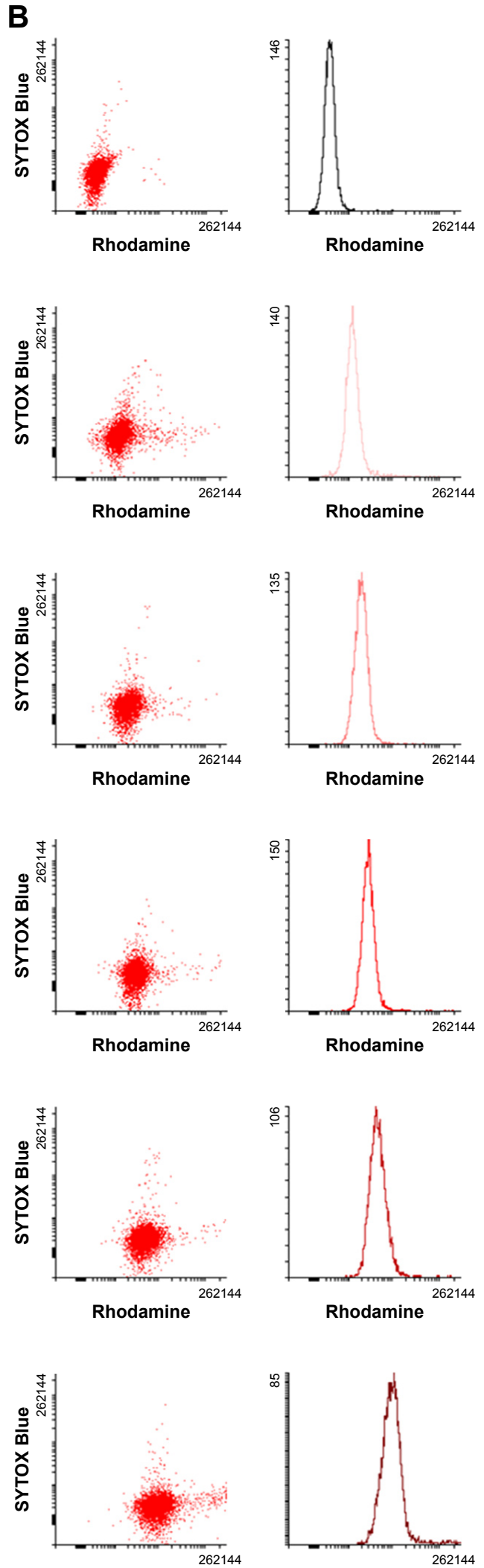

Rhodamine

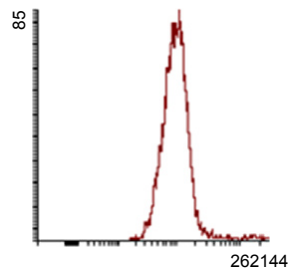

Rhodamine

Figure SI Cellular uptake and cytotoxicity of (A) RhoPro and (B) Rho in HT-29 cells determined through FACS analysis. Dual-wavelength FACS scattergrams showing uptake of the agents and cytotoxicity measured by the intensity of SYTOX Blue and time course of RhoPro uptake by single-channel FACS ( 0 , 5 , I5, 30, 60 , and I20 min). Abbreviations: FACS, fluorescence-activated cell sorting; Rho, rhodamine; RhoPro, rhodamine-protamine. 


\section{Publish your work in this journal}

The International Journal of Nanomedicine is an international, peerreviewed journal focusing on the application of nanotechnology in diagnostics, therapeutics, and drug delivery systems throughout the biomedical field. This journal is indexed on PubMed Central, MedLine, CAS, SciSearch $\AA$, Current Contents ${ }^{\circledR} /$ Clinical Medicine,
Journal Citation Reports/Science Edition, EMBase, Scopus and the Elsevier Bibliographic databases. The manuscript management system is completely online and includes a very quick and fair peer-review system, which is all easy to use. Visit http://www.dovepress.com/ testimonials.php to read real quotes from published authors.

Submit your manuscript here: http://www.dovepress.com/international-journal-of-nanomedicine-journal 